\title{
Methotrexate for Primary Biliary Cirrhosis: Who Is to Be Trusted?
}

\author{
Carlo Selmi · Mauro Podda
}

Published online: 14 October 2010

(C) Springer Science+Business Media, LLC 2010

Primary biliary cirrhosis (PBC) remains a puzzling disease. Its etiology recognizes both genetic and environmental influences, as well epitomized by the incomplete concordance rate among monozygotic twins [1]. Most recently, genomewide association studies provided a number of candidate genes which, however, are found only in subgroups of patients [2]. In a complementary fashion, several environmental factors have also been identified as putative triggers of PBC [3]. Nevertheless, numerous enigmas remain in $\mathrm{PBC}$ pathogenesis and clinical features. Among the latter, poor response of PBC to immunosuppressants, despite evidence supporting the immune-mediated injury and the proinflammatory cascade, remains a clinical challenge. Ursodeoxycholic acid (UDCA) is currently the only accepted treatment for PBC and may delay but not halt the progression of the disease [4]. A few studies suggested increased transplant-free survival, but a meta-analysis of published trials did not confirm this finding [5]. Interestingly, UDCA has limited immunomodulatory effects while acting on biliary secretion and protecting from the potentially membranolytic detergent effect of retained endogenous bile acids [6]. For these reasons, the combination of UDCA and immunosuppressants appeared an obvious and rational development but substantially failed for most drugs [7], while longer study periods are warranted for recent candidates such as budesonide [8], B cell depleting rituximab [9], and farnesoid $\mathrm{X}$ receptor agonist obeticholic acid [10]. The study by Dr. Kaplan and Colleagues

C. Selmi $(\bowtie) \cdot$ M. Podda

Department of Internal Medicine and Hepatobiliary

Immunopathology Unit, IRCCS-Istituto Clinico Humanitas,

University of Milan, via A. Manzoni 56,

20089 Rozzano,

Milan, Italy

e-mail: carlo.selmi@unimi.it included in this issue of Digestive Diseases and Sciences [11] raises some doubt against these quite established observations.

All randomized clinical trials designed to evaluate proposed medical treatments in PBC are hampered by major difficulties in establishing long-term benefits, as the disease manifests a widely variable natural history which may span several decades. Furthermore, surrogate markers of PBC prognosis are scarcely predictive, especially at earlier stages [12], while signs of liver failure are not observed until very late. Similarly, limited predictivity, sampling errors, and inherent invasiveness of liver biopsy limit the role of histology. When immunosuppressants were tested, some efficacy was observed for immunosuppressive treatments including azathioprine, cyclophosphamide, chlorambucil, and prednisone, but their potential toxicity discouraged further studies after the initial ones. An arbitrary representation of the efficacy and safety of the proposed immunomodulatory treatments is presented in Table 1. Apart from ursodeoxycholic acid (UDCA) and the agents currently undergoing confirmation studies, no drug shows a favorable margin in the ratio between efficacy and toxicity with a narrow therapeutic index.

Methotrexate (MTX) is a substantially different story. After preliminary encouraging data coming from a pilot study [13], others reported disappointing results. The beneficial effects of MTX in the treatment of PBC, alone or in combination with UDCA, could not be confirmed by randomized clinical trials performed by other groups [7, $14,15]$. It should be noted, however, that substantial sideeffects were not observed in these studies, probably due also to low doses [7]. Finally, a recent meta-analysis reported no improvement in transplant-free survival or other clinical end-points, albeit with less severe and consistent adverse events [16]. 
Table 1 Efficacy and toxicity of immunosuppressive drugs investigated for medical treatment of primary biliary cirrhosis

\begin{tabular}{lll}
\hline & Efficacy & Adverse events \\
\hline Chlorambucil & \pm & + \\
Cyclosporine & \pm & + \\
Azathioprine & \pm & + \\
Glucocorticoids & \pm & \pm \\
UDCA & + & - \\
Budesonide & + & + \\
Rituximab $^{\text {a }}$ & \pm & \pm \\
Obeticholic acid (FXR agonist) $^{\mathrm{a}}$ & + & -
\end{tabular}

Budesonide, rituximab, and obeticholic acid were only tested in combination with UDCA

a Study results have not been published in extenso

A different approach to identify a potential role for MTX in PBC has been taken in the study by Kaplan et al. [11]. This article does not report data from a randomized clinical trial but rather from the large series and widely recognized clinical expertise of Dr. Kaplan, who first identified poor responders to UDCA then added MTX. The results from serial evaluations include surprisingly favorable effects on liver histology, including fibrosis. Of note, the subgroup of patients with incomplete response to UDCA had no distinguished clinical features, although we noted a higher prevalence of interface hepatitis at histology [17]. The study has substantial advantages that maximize the importance of the reported data. First, the authority represented by the senior author and the group of enthusiast investigators cannot be overlooked. Second, the long observation period and the large number of cases included also strengthen the data. Third and foremost, the treatment algorithm that was developed is rational and quite similar to the decision-making process of clinical practice in the real world. As Dr. Alvan Feinstein suggested, "despite the magnificent scientific achievements of randomized clinical trials, the foundation for a basic science of patient care will also require major attention to the events and observations that occur in the ordinary circumstances of clinical practice" [18]. Nevertheless, the present study has some major limitations which include the retrospective and uncontrolled design, quite correctly defined by the authors as "inescapable."

Who is to be trusted at the end of the day in our clinical practice? Should we include MTX in our armamentarium to treat patients with PBC? Probably both randomized clinical trials and Dr. Kaplan's clinical judgment share part of the truth, but the truth itself may lean more towards one side. Only a well-designed multiphase controlled study of long duration will provide the answer, which could be surprising as in a recent report on liver damage in a large number of patients taking MTX [19] and in light of the frequent PBC rheumatological comorbidities [20]. We may foresee that this will not be an easy challenge, while we expect new treatments based on new molecular targets such as interleukin modulators [21] or antisense microRNAs [22] to be on the horizon soon.

\section{References}

1. Selmi C, Mayo MJ, Bach N, et al. Primary biliary cirrhosis in monozygotic and dizygotic twins: genetics, epigenetics, and environment. Gastroenterology. 2004;127:485-492.

2. Liu X, Invernizzi P, Lu Y, et al. Genome-wide meta-analyses identify three loci associated with primary biliary cirrhosis. Nat Genet. 2010;42:658-660.

3. Selmi C, Gershwin ME. The role of environmental factors in primary biliary cirrhosis. Trends Immunol. 2009;30:415-420.

4. Gong Y, Huang ZB, Christensen E, Gluud C. Ursodeoxycholic acid for primary biliary cirrhosis. Cochrane Database Syst Rev. 2008:CD000551.

5. Poupon RE, Lindor KD, Cauch-Dudek K, et al. Combined analysis of randomized controlled trials of ursodeoxycholic acid in primary biliary cirrhosis. Gastroenterology. 1997;113: 884-890.

6. Paumgartner G, Beuers U. Ursodeoxycholic acid in cholestatic liver disease: mechanisms of action and therapeutic use revisited. Hepatology. 2002;36:525-531.

7. Combes B, Emerson SS, Flye NL, et al. Methotrexate (mtx) plus ursodeoxycholic acid (udca) in the treatment of primary biliary cirrhosis. Hepatology. 2005;42:1184-1193.

8. Rautiainen H, Karkkainen P, Karvonen AL, et al. Budesonide combined with udca to improve liver histology in primary biliary cirrhosis: a three-year randomized trial. Hepatology. 2005;41: 747-752.

9. Myers RP, Shaheen AA, Swain MG, et al. Rituximab for primary biliary cirrhosis (pbc) refractory to ursodeoxycholic acid (udca). Hepatology. 2007;46:550A.

10. Mason A, Luketic V, K.D. L, et al. Farnesoid-x receptor agonists: A new class of drugs for the treatment of pbc? An international study evaluating the addition of int-747 (obeticholic acid) to ursodeoxycholic acid. J Hepatol. 2010;52:S1.

11. Kaplan MM, Bonder A, Ruthazer R, Bonis PAL. Methotrexate in patients with primary biliary cirrhosis who respond incompletely to treatment with ursodeoxycholic acid. Dig Dis Sci. 2010. doi: 10.1007/s10620-010-1291-5.

12. Mayo MJ. Natural history of primary biliary cirrhosis. Clin Liver Dis. 2008;12:277-288. (viii).

13. Kaplan MM, Knox TA. Treatment of primary biliary cirrhosis with low-dose weekly methotrexate. Gastroenterology. 1991;101: 1332-1338.

14. Hendrickse MT, Rigney E, Giaffer MH, et al. Low-dose methotrexate is ineffective in primary biliary cirrhosis: long-term results of a placebo-controlled trial. Gastroenterology. 1999;117: 400-407.

15. Gonzalez-Koch A, Brahm J, Antezana C, Smok G, Cumsille MA. The combination of ursodeoxycholic acid and methotrexate for primary biliary cirrhosis is not better than ursodeoxycholic acid alone. J Hepatol. 1997;27:143-149.

16. Giljaca V, Poropat G, Stimac D, Gluud C. Methotrexate for primary biliary cirrhosis. Cochrane Database Syst Rev. 2010;5: CD004385. 
17. Chazouilleres O, Wendum D, Serfaty L, et al. Primary biliary cirrhosis-autoimmune hepatitis overlap syndrome: clinical features and response to therapy. Hepatology. 1998;28:296-301.

18. Feinstein AR. An additional basic science for clinical medicine: Ii. The limitations of randomized trials. Ann Intern Med. 1983;99:544-550.

19. Quintin E, Scoazec JY, Marotte H, Miossec P. Rare incidence of methotrexate-specific lesions in liver biopsy of patients with arthritis and elevated liver enzymes. Arthritis Res Ther. 2010; 12:R143.
20. Selmi C, De Santis M, Gershwin ME. Liver involvement in subjects with rheumatic disease. Arthritis Res Ther. 2010 (in press).

21. Yoshida K, Yang GX, Zhang W, et al. Deletion of interleukin12 p40 suppresses autoimmune cholangitis in dominant negative transforming growth factor beta receptor type ii mice. Hepatology. 2009;50:1494-1500.

22. Padgett KA, Lan RY, Leung PC, et al. Primary biliary cirrhosis is associated with altered hepatic microrna expression. J Autoimmun. 2009;32:246-253. 